\title{
Anticoncepción de emergencia en estudiantes mexicanos
}

\author{
Ahidee Leyva-López, M en C, (') Rubén Chávez-Ayala, M en Psicol, ${ }^{(1)}$ Erika EAtienzo, M en C, ${ }^{(1)}$ \\ Betania Allen-Leigh, D en Antrop,(l) Dolores Ramírez-Villalobos, M en C, (I) \\ Elsa Yunes-Díaz, M en C, ${ }^{(1)}$ Leonor Rivera-Rivera, M en C. ${ }^{(1)}$
}

Leyva-López A, Chávez-Ayala R,Atienzo EE,Allen-Leigh B, Ramírez-Villalobos D, Yunes-Díaz E, Rivera-Rivera L. Anticoncepción de emergencia en estudiantes mexicanos. Salud Publica Mex 2010;52:156-164.

\section{Resumen}

Objetivo. Evaluar el conocimiento y uso de anticoncepción de emergencia (AE), y factores asociados en estudiantes de escuelas públicas en Morelos, México. Material y métodos. Estudio transversal realizado entre 2003-2004 con I 550 estudiantes de 14-24 años. Se utilizó regresión logística para identificar factores asociados al conocimiento correcto sobre AE. Resultados. El $40 \%$ fueron hombres y $60 \%$ mujeres con edad promedio de 19 años. El $60 \%$ ha escuchado sobre $\mathrm{AE}$ pero únicamente $45 \%$ tiene conocimiento correcto; $3 \%$ de los estudiantes sexualmente activos la ha usado. Factores asociados al conocimiento correcto: ser mujer $(R M=1.66$; IC I.33, 2.06), haber tenido relaciones sexuales $(R M=I .77 ;$ IC I.43, 2. I8), nivel socioeconómico medio $(R M=1.87$; IC I.04, $3.37)$, vivir en zona semi-urbana $(R M=1.34$; $I C I .02,1.77)$, tomar alcohol $(R M=I .78 ; I C I .42,2.22)$. Conclusiones. En Morelos, el conocimiento de los adolescentes sobre la AE es escaso. La transmisión de mensajes claros sobre su forma precisa de uso resulta necesaria.

Palabras clave: anticoncepción postcoital; adolescentes; estudiantes; conocimiento; México
Leyva-López A, Chávez-Ayala R,Atienzo EE,Allen-Leigh B, Ramírez-Villalobos D,Yunes-Díaz E, Rivera-Rivera L. Emergency contraception in Mexican students. Salud Publica Mex 2010;52:156-164.

\section{Abstract}

Objective. To evaluate emergency contraception (EC) knowledge and use as well as identify factors associated to correct EC knowledge among public schools students in Morelos, Mexico. Material and Methods. Cross-sectional study conducted in 2003-2004 in a sample of I 550 students (ages I4-24). Logistic regression was used to identify factors associated to EC correct knowledge. Results. Among all, $40 \%$ were men and $60 \%$ women with an average age of 19 years. Sixty percent have heard about EC, nevertheless, only $45 \%$ have correct knowledge about it; $3 \%$ of sexually active students $(43 \%)$ reported its use. Factors associated to EC correct knowledge are: being a woman $(O R=1.66 ; \mathrm{Cl} I, 33$, $2,06)$, being sexually active $(O R=I .77 ; C l I, 43,2,18)$, middle income $(O R=I .87 ; C l I, 04,3,37)$, living in a semi-urban zone $(O R=1.34 ; C l I, 02, I, 77)$, drinking alcohol $(O R=1.78 ; C l$ I,42, 2,22). Conclusions. In Morelos, adolescents' knowledge of $\mathrm{EC}$ is scarce. Specific messages regarding its correct use are necessary.

Keywords: postcoital contraception; adolescents; students; knowledge; Mexico

(I) Instituto Nacional de Salud Pública. Cuernavaca, Morelos, México.

Fecha de recibido: II de junio de 2009 • Fecha de aceptado: 29 de octubre de 2009

Solicitud de sobretiros: Mtra. Leonor Rivera Rivera. Dirección de Salud Reproductiva, Centro de Investigación en Salud Poblacional, Instituto Nacional de Salud Pública. Av. Universidad 655, col. Santa María Ahuacatitlán. 62 I00, Cuernavaca, Morelos, México.

Correo electrónico: Irivera@correo.insp.mx 
R ecientemente la anticoncepción de emergencia (AE) ha sido incluida en la Norma Oficial Mexicana de Servicios de Planificación Familiar (NOMSSA2-005-1993), ${ }^{1}$ y en el Cuadro Básico de Medicamentos de la Secretaría de Salud. ${ }^{2,3}$ Sin embargo, proveer a los / las adolescentes de información concreta sobre su uso adecuado entraña grandes retos para los programas de salud sexual y reproductiva, especialmente por las controversias generadas en torno a este método.

La AE, llamada así por tratarse de un método para la planificación familiar (PF) que no es de uso cotidiano, 4-7 puede utilizarse inmediatamente o hasta cinco días después en caso de uso incorrecto o falla de un método anticonceptivo, o debido a una relación sexual forzada (violación). ${ }^{8,9}$ Esta constituye una valiosa oportunidad como respaldo anticonceptivo postcoital por ser un método simple, seguro y efectivo ${ }^{1,6}$ que pueden utilizar las mujeres para prevenir un embarazo no deseado. A pesar de indicaciones por grupos de oposición, la AE no tiene propiedades para interrumpir el embarazo. ${ }^{10,11}$

En Estados Unidos, la Administración de Alimentos y Drogas autorizó la venta libre de AE a mujeres mayores de 18 años. ${ }^{12}$ No obstante, algunos reportes se enfrentan a dificultades con respecto a su acceso porque existe creencia que son métodos abortíferos o espermicidas tóxicos para el organismo. Ante esto, los/las adolescentes pueden sentirse avergonzados de acudir y solicitar este método en una clínica de PF. ${ }^{13,14}$

México es un país heterogéneo, de ingreso medio ${ }^{15}$ y con 104.9 millones de habitantes, ${ }^{16}$ de los cuales, aproximadamente 21 millones son jóvenes entre 15-24 años. ${ }^{17,18} \mathrm{~A}$ pesar de esfuerzos dirigidos a reducir embarazos en este grupo, disminuir la tasa de fertilidad es un difícil reto de alcanzar. Incluso, estimaciones de la Encuesta Nacional de Salud y Nutrición en México 2006 indican que 695100 mujeres entre 12-19 años han estado embarazadas alguna vez. ${ }^{19}$ En Morelos, $0.36 \%$ de todos los nacimientos registrados en 2004 correspondieron a mujeres menores de 15 años, mientras que 15.38 y $28.15 \%$ se presentaron en mujeres de $15-19$ y de 20-24 años respectivamente. ${ }^{20}$ En el mismo estado otro estudio encontró que en cada una de cinco madres adolescentes su embarazo no fue deseado. ${ }^{5}$ Lo anterior conlleva a reflexionar sobre las prácticas anticonceptivas de los/ las adolescentes en México y las implicaciones sobre su salud. Estadísticas recientes señalan que entre jóvenes de 12-19 años que han iniciado vida sexual, 29.6\% de hombres y $56.6 \%$ de mujeres no utilizaron ningún método anticonceptivo en su primera relación sexual. ${ }^{19}$ En este contexto, si bien la $\mathrm{AE}$ representa un oportuno método de respaldo, según la Encuesta Nacional de Juventud $2005^{21}$ sólo $35.9 \%$ de los/las adolescentes conoce sobre
$\mathrm{AE}$, y entre éstos, únicamente $12.7 \%$ reporta haberla utilizado alguna vez.

Promover el conocimiento y uso de la AE como derecho sexual y reproductivo es de excepcional importancia porque amplía las posibilidades de decidir de manera libre, responsable e informada sobre el número de hijos a tener. ${ }^{22-24}$ No obstante, se vuelve indispensable identificar aquellos factores que influyen de manera positiva en la adquisición de información adecuada entre los/las adolescentes.

Así, en el presente estudio se evaluará el nivel de conocimiento y uso de $\mathrm{AE}$, así como factores asociados al conocimiento correcto en estudiantes de escuelas públicas en Morelos, México, entre 2003-2004.

\section{Material y métodos}

\section{Diseño y población de estudio}

Se desarrolló un estudio transversal utilizando datos provenientes de la tercera medición de una cohorte de estudiantes de escuelas públicas de Morelos, México. ${ }^{25}$ En la medición basal se usó un diseño de muestreo por conglomerados que generó muestras representativas de estudiantes de acuerdo al grado escolar, y donde las escuelas se seleccionaron con una probabilidad proporcional a la matrícula de estudiantes. El estudio recibió la aprobación de los Comités de Ética y de Investigación del Instituto Nacional de Salud Pública.

Para fines del estudio mediante un muestreo por conveniencia se obtuvo una submuestra de 92 escuelas de nivel medio superior (1o, 20 y 3er grado de preparatoria), tres universidades y tres institutos tecnológicos (ambos de 1er grado) y se invitó al estudio a estudiantes de 14-24 años que participaron en la medición basal y que durante 2003-2004 aún se encontraban registrados en dichas escuelas. Se utilizó ésta medición debido a que fue la única que contenía información sobre la $\mathrm{AE}$.

En total participaron 1719 estudiantes, sin embargo, 169 fueron excluidos del análisis porque los cuestionarios respondidos carecían de información sobre edad, escolaridad, haber tenido o no relaciones sexuales, y conocimiento y uso de métodos anticonceptivos. Finalmente la muestra de estudio se acotó a 1550 estudiantes.

Cabe mencionar que antes de que los estudiantes contestaran en su escuela el cuestionario autoaplicado, firmaron una carta de consentimiento informado. Cuando se trató de un menor de edad, se solicitó a padres o tutores consentimiento para que sus hijos(as) participaran. En ambos casos, se explicó en detalle objetivos del estudio, confidencialidad y contenido del cuestionario 
que contenía las secciones: a) factores sociodemográficos, b) factores familiares, c) factores psicológicos, d) violencia intrafamiliar, y e) comportamiento sexual. Para fines de este estudio, únicamente se analizaron las secciones $a, b, c$ y e.

\section{Medición de las variables}

La variable dependiente de este estudio fue conocimiento correcto sobre $A E(C A E)$. Para medir esta variable se utilizaron dos reactivos. Primero se preguntó a los estudiantes: ¿Qué son los AE?, con las siguientes opciones de respuesta: a) un método de aborto, b) pastillas que tomas después de tener relaciones para prevenir un embarazo, c) pastillas que te pones en la vagina para prevenir embarazo antes de las relaciones sexuales, d) pastillas que te pones en la vagina para prevenir embarazo después de las relaciones sexuales y e) no sé. Se consideró respuesta correcta cuando un estudiante respondió la opción b y respuesta incorrecta cuando se respondió a, c, d o e. También se preguntó: ¿Cuánto tiempo después de tener relaciones sexuales sin protección puedes utilizar AE? con las opciones: a) 12 horas, b) 1 día, c) 2 días, d) 3 días y e) no sé. Se consideró respuesta correcta las opciones $\mathrm{a}, \mathrm{b}$, c o d y respuesta incorrecta a la opción e. Posteriormente se generó la variable dicotómica CAE considerando conocimiento correcto cuando un estudiante respondió correctamente a las dos preguntas previamente descritas. Además, se preguntó de manera general si alguna vez habían escuchado hablar sobre la AE.

Las variables independientes de interés fueron: variables sociodemográficas: sexo, edad, estado civil, ocupación, zona rural/urbana, tipo de escuela y nivel socioeconómico (NSE). Para el NSE se construyó un índice descrito por Bronfman; ${ }^{26}$ variables familiares: nivel educativo y antecedente de consumo de drogas de los padres, éste último medido con la pregunta: ¿Tu padre/ madre usa marihuana como droga? con respuestas: 1) sí y 2) no; variables psicológicas: autoestima, medida con el inventario de Coopersmith, donde autoestima se define como la percepción y evaluación que el sujeto hace con respecto a sí mismo, ${ }^{27}$ y antecedentes de consumo de drogas, alcohol y tabaco con el antecedente de drogas medido por la pregunta: $¿$ Te inyectas o inhalas algún tipo de droga? Con respuestas: 1) sí y 2) no. En caso afirmativo, marcaban cocaína, cemento, morfina, hongos, marihuana, thiner, chochos, entre otros; se consideró bebedor o fumador al estudiante que reconoció haber consumido alcohol o tabaco aunque fuera en ocasiones. La última variable fue comportamiento sexual (inicio de relaciones sexuales, motivo, edad de inicio, número de relaciones y de parejas sexuales, y uso de anticonceptivos).

\section{Análisis de la información}

Se realizó un análisis descriptivo para cada variable para conocer su distribución y detectar valores fuera de rango. Posteriormente, se realizaron análisis bivariados con pruebas de ji cuadrada. Para el análisis multivariado se construyeron modelos de regresión logística múltiple saturados y se ajustó por efectos del diseño de la encuesta, esto es, un muestreo por conglomerados. Para el modelo final se identificaron variables que mostraron asociación con CAE considerando un valor $p \leq 0.05$. La bondad de ajuste del modelo se evaluó mediante la prueba de Hosmer-Lemeshow. ${ }^{28}$ Para todos los análisis estadísticos se utilizó el programa Stata v9.0.*

\section{Resultados}

\section{Características sociodemográficas}

Entre los 1550 estudiantes, la edad promedio fue 19 \pm 2 años. Hubo mayor proporción de mujeres (60.19\%) en comparación con los hombres. La mayoría de los estudiantes fueron solteros/as (93.4\%) y más de la mitad señaló que solamente se dedicaba a estudiar (63\%). En relación con el NSE, 89.1\% se concentró en un nivel medio (cuadro I). Al comparar estas características con estudiantes que fueron excluidos del estudio, se puede decir que ambos grupos son similares: la edad promedio fue de $20 \pm 2$ años, siendo la mayoría solteros $(92.17 \%)$, sin otra ocupación más que estudiante $(87.85 \%)$ y NSE medio $(62.65 \%)$.

\section{Comportamiento sexual y uso de AE}

Las características en relación con el comportamiento sexual de los estudiantes se muestran en el cuadro II. Del total de estudiantes participantes $43.2 \%(n=670)$ reportó haber tenido relaciones sexuales alguna vez; sin embargo, tal y como era esperado esta proporción varió según el nivel educativo: $23.48 \%$ de preparatoria y $19.75 \%$ de nivel superior indicaron relaciones sexuales. Entre el sub-grupo de estudiantes sexualmente activos la media de edad de su primera relación sexual (prelsex) fue $19.5 \pm 2$ años. La mayoría señaló haber tenido la prelsex con su novio/a (89.4\%), argumentando el hecho de sentir amor por él/ ella (32.84\%); no obstante, resulta importante destacar que sólo la mitad señaló sentirse feliz o satisfecho/a después de dicha relación (54.33\%).

\footnotetext{
* Stata Corporation, 4905 Lakeway Drive Special Edition College Station, Texas 77845 USA 800-STATA-PC.
} 
Cuadro I

\section{Características sociodemográficas de estudiantes de escuelas públicas de Morelos, México, SEGÚN SEXO Y ANTECEDENTE DE RELACIONES SEXUALES, 2003-2004}

\begin{tabular}{|c|c|c|c|c|c|}
\hline & & \multicolumn{4}{|c|}{$n=1550$} \\
\hline & & \multicolumn{2}{|c|}{ Hombres $(n=6 \mid 7)$} & \multicolumn{2}{|c|}{ Mujeres $(n=933)$} \\
\hline & & $\begin{array}{c}\text { Sin inicio de vida } \\
\text { sexual }(n=326) \%\end{array}$ & $\begin{array}{l}\text { Con inicio de vida } \\
\text { sexual }(n=29 I) \%\end{array}$ & $\begin{array}{l}\text { Sin inicio de vida } \\
\text { sexual }(n=379) \%\end{array}$ & $\begin{array}{l}\text { Con inicio de vida } \\
\text { sexual }(n=554) \%\end{array}$ \\
\hline \multirow[t]{4}{*}{ Edad } & $14-17$ & 13.50 & 25.77 & 29.60 & 21.64 \\
\hline & $18-19$ & 23.62 & 28.87 & 34.66 & 28.23 \\
\hline & $20-21$ & 34.66 & 23.37 & 21.48 & 26.39 \\
\hline & $22-24$ & 28.22 & 21.99 & 14.26 & 23.75 \\
\hline \multirow[t]{3}{*}{ Tipo de escuela } & Preparatoria & 57.98 & 49.48 & 54.15 & 58.05 \\
\hline & Universidad & 30.06 & 34.02 & 32.49 & 30.34 \\
\hline & Nivel medio superior & 11.96 & 16.49 & 13.36 & $|11.6|$ \\
\hline \multirow[t]{3}{*}{ Estado civil } & Soltero/a & 93.87 & 96.22 & 92.06 & 92.88 \\
\hline & Casado/a, unión libre & 3.68 & 3.09 & 5.78 & 5.28 \\
\hline & Divorciado(a)/separado(a) & 2.45 & 0.69 & 2.17 & 1.85 \\
\hline \multirow[t]{3}{*}{ Ocupación } & Estudia & 62.27 & 59.79 & 63.0 & 68.87 \\
\hline & Estudia y trabaja & 2.45 & 3.78 & 3.07 & 3.17 \\
\hline & Otro & 35.28 & 36.43 & 33.94 & 27.97 \\
\hline \multirow[t]{3}{*}{ NSE* } & Bajo & 6.75 & 3.78 & 2.71 & 3.17 \\
\hline & Medio & 87.12 & 87.97 & 91.70 & 87.86 \\
\hline & Alto & 6.13 & 8.25 & 5.60 & 8.97 \\
\hline \multirow[t]{3}{*}{ Tipo de zona de residencia } & Rural & 36.20 & 39.52 & 30.51 & 33.25 \\
\hline & Semi-urbana & 26.38 & 20.62 & 27.08 & 26.39 \\
\hline & Urbana & 37.42 & 39.86 & 42.42 & 40.37 \\
\hline
\end{tabular}

Caracteristicas familiares

Escolaridad del padre

Ninguno 5.21

Primaria incompleta 26.38

Primaria completa 25.46

Secundaria o más $\quad 42.94$

$\begin{array}{lll}6.19 & 7.40 & 6.07\end{array}$

24.05

43.64

29.78

27.44

Ninguno 6.13

Primaria incompleta

28.83

Primaria completa

28.53

Secundaria o más

36.50

7.90

29.55

30.58

31.96

35.38

26.39

Secundaria o más

Consumo de drogas en los padres

$\begin{array}{lr}\text { No } & 97.85 \\ \text { Sí } & 2.15\end{array}$

2.15

90.38

9.62

1.81

$6.32 \quad 9.23$

$33.94 \quad 29.29$

$29.06 \quad 29.55$

$\begin{array}{ll}0.69 & 31.95\end{array}$

Características psicosociales

\begin{tabular}{|c|c|c|c|c|c|}
\hline Fuma & No & 65.64 & 64.95 & 76.90 & 78.63 \\
\hline & Sí & 34.36 & 35.05 & 23.10 & 21.37 \\
\hline \multirow[t]{2}{*}{ Consumo de bebidas alcohólicas } & No & 45.09 & 49.48 & 32.67 & 31.40 \\
\hline & Sí & 54.91 & 50.52 & 67.33 & 68.60 \\
\hline \multirow[t]{2}{*}{ Autoestima } & Bajo & 63.80 & 61.86 & 61.01 & 65.96 \\
\hline & Alto & 36.20 & 38.14 & 38.99 & 34.04 \\
\hline \multirow[t]{2}{*}{ Locus de control interno } & Bajo & 1.57 & 1.43 & 2.44 & 3.04 \\
\hline & Alto & 98.43 & 98.57 & 97.56 & 96.96 \\
\hline \multirow[t]{2}{*}{ Locus de control externo } & Bajo & 43.93 & 50.18 & 47.17 & 46.87 \\
\hline & Alto & 56.07 & 49.82 & 52.83 & 53.13 \\
\hline
\end{tabular}

*NSE= nivel socioeconómico 


\section{Cuadro II}

\section{Comportamiento sexual de estudiantes de escuelas públicas de Morelos, México 2003-2004}

\begin{tabular}{|c|c|c|c|}
\hline \multirow[b]{3}{*}{ Con quién tuvo su primera relación sexual } & \multicolumn{3}{|c|}{$n=670$} \\
\hline & Hombres $n=291 \%$ & Mujeres $n=37 \%$ & Valor "p"* \\
\hline & & & \\
\hline Novio/a & 37.31 & 52.09 & \\
\hline Amigo/a & 2.99 & 3.58 & 0.00 \\
\hline Otro & 1.64 & 2.59 & \\
\hline Edad en la primera relación sexual & & & \\
\hline $14-17$ & 21.64 & 1.19 & \\
\hline$|8-2|$ & 49.10 & 4.78 & 0.58 \\
\hline $22-24$ & 21.34 & 1.64 & \\
\hline Por qué tuvo su primera relación sexual & & & \\
\hline Por amor & 15.07 & 17.76 & \\
\hline Por curiosidad & 14.03 & 18.21 & 0.44 \\
\hline Por deseo & 10.30 & 16.27 & \\
\hline Por satisfacer a la pareja & 4.03 & 4.33 & \\
\hline ¿Cómo se sintió después de su primera relación sexual? & & & \\
\hline Alegre y satisfecho/a & 27.01 & 27.31 & 0.00 \\
\hline Con miedo, culpable, arrepentido/a, con coraje o avergonzado/a & 11.49 & 19.70 & \\
\hline Ninguna emoción en particular & 4.93 & 9.55 & \\
\hline Usó algún método anticonceptivo en la primera relación sexual & & & \\
\hline No & 33.28 & 40.45 & 0.13 \\
\hline Sí & 10.15 & 16.12 & \\
\hline Método que usó en la primera relación sexual $\left.\right|^{\S}$ & & & \\
\hline Condón & 23.30 & 30.68 & \\
\hline Pastillas o inyecciones & 2.84 & 2.27 & \\
\hline Retirarse o ritmo & 1.14 & 2.27 & 0.63 \\
\hline Anticoncepción de emergencia & 1.14 & 2.27 & \\
\hline No dijo cuál & 10.23 & 23.86 & \\
\hline
\end{tabular}

Frecuencia con la que usa algún método anticonceptivo para evitar un embarazo o alguna infección de transmisión sexual

Siempre $\quad 12.24$

Siempre

14.18

11.19

Casi siempre

8.36

18.81

A veces

8.66

14.93

Nunca

11.64

Método anticonceptivo utilizado en su última relación sexual

$\begin{array}{lrr}\text { Condón } & 26.57 & 34.03 \\ \text { Pastillas, óvulos } & 3.73 & 4.63 \\ \text { Anticoncepción de emergencia } & 0.10 & 0.20 \\ \text { Ninguno } & 13.13 & 17.61\end{array}$

*Valor p para la diferencia de proporciones utilizando la prueba de $j^{2}$

$\S$ De aquellos que sí usaron método en la primera relación sexual 
Por otro lado, el uso de algún método anticonceptivo durante la prelsex fue $26.27 \%$ (10.15\% hombres y $16.12 \%$ mujeres); entre éstos, el principal método utilizado fue el condón (53.98\%). En contraste, el uso de la AE fue reportado únicamente en $3.4 \%$, con $1 \%$ hombres y $2.3 \%$ mujeres respectivamente.

\section{Conocimiento sobre métodos anticonceptivos y factores asociados al conocimiento correcto sobre AE}

Cuando se preguntó a estudiantes qué métodos anticonceptivos conocían, la mayoría respondió principalmente que el condón (97.48\%), seguido de pastillas $(92.65 \%$ ) y de vasectomía $(76.77 \%)$, mientras que el método menos reportado fue el de Billings (12.45\%). Por otro lado, $60 \%$ de todos los estudiantes ha escuchado hablar alguna vez sobre la $\mathrm{AE}$, sin embargo sólo $45 \%$ tiene $\mathrm{CAE}$ correcto (figura 1). Destaca el hecho de que es menor la proporción de hombres que tiene CAE correcto (39\%) en comparación con mujeres (49\%), con diferencias estadísticamente significativas. Por otra parte, al analizar el tipo de escuela se encontró que los participantes de preparatorias tienen $26.32 \%$ CAE correcto, mientras que aquellos de universidades e institutos tecnológicos tiene solamente 13.03 y $5.74 \%$ respectivamente.

El cuadro III muestra razones de momios crudas y ajustadas por las variables que ahí se presentan. Asimismo, el análisis multivariado señala que los factores

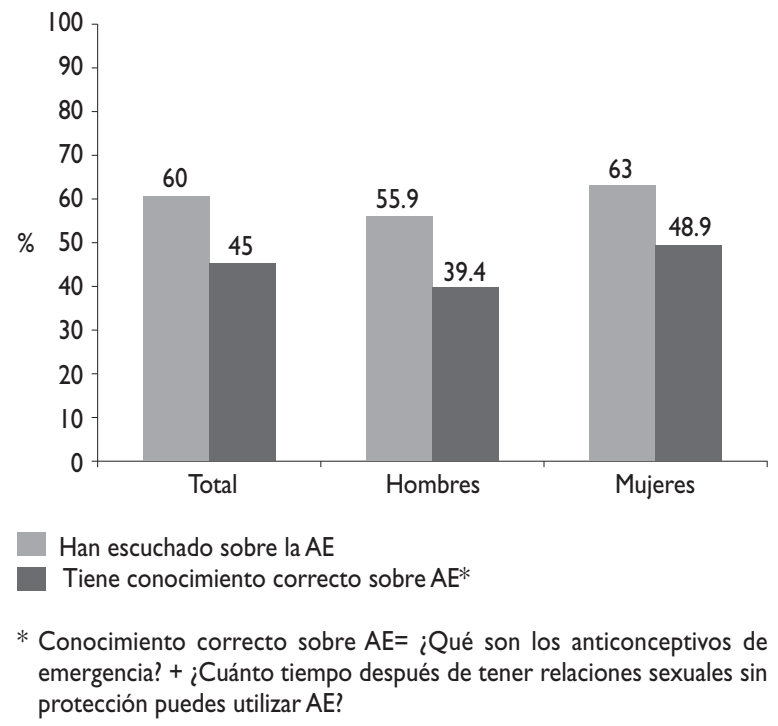

Figura I. Conocimiento sobre anticoncepción de EMERGENCIA SEGÚN SEXO DE LOS ESTUDIANTES EN ESCUELAS públicas de Morelos, México, 2003-2004 asociados positivamente al CAE fueron: ser mujer, haber tenido relaciones sexuales, NSE medio, pertenecer a una zona semi-urbana/urbana, tomar bebidas alcohólicas y tener padres con antecedente de uso de drogas. Por el contrario, provenir de una escuela tecnológica redujo las posibilidades de identificar correctamente qué es la $\mathrm{AE}$. A pesar de que la variable de autoestima no tuvo asociación estadísticamente significativa con CAE, se incluyó en el modelo final porque juega un rol mediador entre los factores asociados al CAE. La prueba de bondad de ajuste de Hosmer-Lemeshow para el modelo final fue de $4.39, p=0.82$, indicando que el modelo muestra asociaciones válidas y estadísticamente significativas. A continuación se discuten las implicaciones de los resultados encontrados en el análisis multivariado.

\section{Discusión}

En México, en los últimos años se ha generado gran debate y polémica en relación con la AE y con su promoción entre los jóvenes. No obstante, los resultados de este estudio indican que si bien gran proporción de estudiantes han escuchado acerca del método, menos de la mitad tiene conocimiento adecuado respecto de su uso. Además, estos resultados aportan evidencia a la creciente literatura médica que demuestra que los adolescentes hombres poseen menor información sobre aspectos básicos para la prevención de embarazos, ${ }^{29-32}$ pues como aquí se reporta, son las mujeres quienes encabezan el porcentaje de un CAE correcto. Lo anterior puede estar relacionado con que en México la construcción social de la salud sexual y reproductiva supone que la responsabilidad del comportamiento reproductivo compete a las mujeres y no a los hombres, limitando así el papel de éstos en la prevención de embarazos. ${ }^{33}$ No obstante, los hombres pueden ocupar un papel crucial como agentes en la promoción de la salud sexual al facilitar la comunicación y negociación con sus parejas respecto del uso de anticonceptivos. ${ }^{34,35}$

Por otro lado, se encontró que los estudiantes de institutos tecnológicos de educación superior identifican correctamente qué es la $\mathrm{AE}$ en menor proporción con respecto de los estudiantes de preparatorias y universidades. Resulta difícil argumentar en relación con este punto, pues para explorar a profundidad este aspecto es necesario la inclusión de otras variables relacionadas con el contexto escolar, sin embargo, se podría suponer que esto es un indicador de que las escuelas mantienen un rol diferente en relación con la difusión de temas sobre prevención entre sus estudiantes o bien, de que el comportamiento y las características de los estudiantes de las distintas escuelas es diferente, incluso podría tratarse de cierto efecto generacional. 


\section{Cuadro III}

Determinantes del CONOCIMIENTO CORRECTO SOBRE LA ANTICONCEPCIÓN DE EMERGENCIA EN ESTUDIANTES de escuelas Públicas de Morelos, México 2003-2004

\begin{tabular}{|c|c|c|c|c|}
\hline$n=I 550$ & RM Cruda & IC $95 \%$ & $R M^{*}$ ajustada & IC $95 \%$ \\
\hline \multicolumn{5}{|l|}{ Sexo } \\
\hline Hombre & 1.00 & & 1.00 & \\
\hline Mujer & 1.47 & $1.19,1.80$ & 1.66 & $1.33,2.06$ \\
\hline \multicolumn{5}{|l|}{ Relaciones sexuales } \\
\hline No & 1.00 & & 1.00 & \\
\hline Sí & I.7I & $1.40,2.10$ & I.77 & $1.43,2.18$ \\
\hline \multicolumn{5}{|l|}{ Ocupación } \\
\hline Estudia & 1.00 & & 1.00 & \\
\hline Estudia y trabaja & 1.72 & $0.95,3.12$ & 1.77 & $0.96,3.28$ \\
\hline Estudia y otra actividad (ama de casa) & 0.76 & $0.61,0.94$ & 0.79 & $0.63,0.99$ \\
\hline \multicolumn{5}{|l|}{ NSE } \\
\hline Bajo & 1.00 & & 1.00 & \\
\hline Medio & 2.12 & $1.20,3.36$ & 1.87 & $1.04,3.37$ \\
\hline Alto & 2.22 & I.I3,4.37 & 1.79 & $0.89,3.60$ \\
\hline \multicolumn{5}{|l|}{ Tipo de zona } \\
\hline Rural & 1.00 & & 1.00 & \\
\hline Semi-urbana & 1.30 & $1.00,1.69$ & 1.34 & $1.02,1.77$ \\
\hline Urbana & 1.21 & $0.96, I .54$ & 1.25 & $0.95,1.63$ \\
\hline \multicolumn{5}{|l|}{ Tipo de escuela } \\
\hline Preparatorias & 1.00 & & 1.00 & \\
\hline Universidades & 0.75 & $0.60,0.95$ & 0.67 & $0.52,0.85$ \\
\hline Escuelas tecnológicas & 0.84 & $0.61,1.37$ & 0.69 & $0.48,0.99$ \\
\hline \multicolumn{5}{|l|}{ Consumo de bebidas alcohólicas } \\
\hline No & 1.00 & & 1.00 & \\
\hline Sí & 1.58 & $1.28, .94$ & 1.78 & $1.42,2.22$ \\
\hline \multicolumn{5}{|c|}{ Antecedente de consumo de drogas por sus padres } \\
\hline No & 1.00 & & 1.00 & \\
\hline Sí & 1.84 & $1.07,3.15$ & 1.78 & $1.02,3.11$ \\
\hline \multicolumn{5}{|l|}{ Autoestima } \\
\hline Bajo & 1.00 & & 1.00 & \\
\hline Alto & 1.02 & $0.83,1.25$ & 1.07 & $0.86,1.33$ \\
\hline
\end{tabular}

Sin duda, este es un aspecto importante a considerar en el momento de identificar factores que inciden positivamente en la adquisición de conocimientos y habilidades útiles en la prevención de embarazos entre los/las adolescentes.
Los resultados en relación con el uso de la $\mathrm{AE}$ merecen especial atención ya que, si bien la proporción de estudiantes que han iniciado relaciones sexuales es relativamente alta, la proporción que reporta hacer uso de este método es notoriamente baja. Esto tiene una im- 
plicación primordial al considerar que muchos de estos estudiantes no utilizaron ningún método anticonceptivo en su primera relación sexual. No se debe perder de vista que la mayoría de los participantes se encontraba cursando estudios de preparatoria, por lo que, si se consideran los riesgos psico-sociales que un embarazo implica en la vida de los / las adolescentes, ${ }^{36}$ el hecho de conocer este método resulta particularmente ventajoso. Aún así, existe evidencia de que los estudiantes conocen más sobre otros métodos que sobre la AE. Con los resultados aquí descritos se delinea de nuevo un entorno en el que existe un riesgo inminente para un embarazo no planeado y también para ITS, pues aún cuando el uso del condón es por mucho más elevado que el de $\mathrm{AE}$, su utilización sigue permaneciendo baja.

Diversos estudios a nivel nacional e internacional han demostrado que la $\mathrm{AE}$ es un método aceptado por la población..$^{30-37}$ Sin embargo, al analizar la baja proporción aquí reportada en el uso de este método, resulta fundamental considerar que en México prevalece una amplia brecha entre el hecho de poseer información sobre los métodos y el hacer un uso efectivo de ellos. ${ }^{38}$ Para lograr que la AE tenga un impacto potencial en la salud reproductiva de los/las adolescentes, se requiere no sólo de su difusión sino también de la construcción de un puente que facilite su acceso.

Cabe destacar que el análisis aquí desarrollado siguió procedimientos metodológicos rigurosos, sin embargo, está sujeto a algunas limitaciones. En primer lugar se encuentra su carácter transversal ${ }^{39} \mathrm{y}$ que los resultados de este estudio son representativos solamente para aquella población estudiantil mexicana con características similares. Asimismo, la información analizada proviene de cuestionarios autoaplicados que conllevan los problemas inherentes al autorreporte. Particularmente, es posible que la baja prevalencia encontrada en relación con el uso de la AE esté sujeta a subreporte debido a los juicios de orden moral que aún existen en relación con la AE. Es también posible que la manera en que se plantearon los reactivos sobre la AE se prestara a alguna confusión entre los estudiantes; al respecto, en años recientes se han desarrollado reactivos sobre $\mathrm{AE}$ más específicos, detallados y claros. ${ }^{40}$

Se reconoce que los reactivos utilizados para evaluar conocimiento sobre $\mathrm{AE}$ se encuentran sujetos a problemas de validez por varios aspectos. En primer lugar se encuentra el hecho de que si bien existen en el mercado distintas prescripciones de $\mathrm{AE}$, la variable utilizada en este estudio hace referencia únicamente a la vía de administración oral sin ser esto especificado en la pregunta. No obstante, las opciones de respuesta incluidas en el cuestionario hacen referencia a las pastillas, por lo que la posibilidad de responder incorrectamente debido a una confusión por el tipo de prescripción es poca. Es decir, queda claro en el cuestionario que la pregunta hace referencia a las pastillas AE. Asimismo, la redacción de la pregunta en relación con el tiempo límite para utilizar la $\mathrm{AE}$ puede ser en sí misma confusa, pues cualquiera de las primeras cuatro opciones de respuesta son correctas, es decir, la AE puede ser utilizada desde las 12 primeras horas después de la relación sexual, hasta tres días después. Al tener esto presente desde el inicio del estudio, la información se analizó de tal forma que cualquiera de estas opciones de respuesta representara CAE. A pesar de estas limitaciones, las estimaciones obtenidas son valiosas, más aún si se considera que al momento del estudio la $\mathrm{AE}$ aún no formaba parte de la NOM-SSA2-005-1993; ; por esta razón es posible que en la actualidad la situación respecto a su difusión y reconocimiento entre los jóvenes sea mayor a la aquí reportada, ya que incluso estudios recientes en México han mostrado un incremento en los últimos años en relación con el conocimiento de la AE. 37,41

Adicionalmente, es importante indicar que aunque en el consumo de drogas de padres únicamente se rescató información sobre el uso de la marihuana, este dato aporta información para tener un CAE correcto.

Finalmente, los resultados del estudio indican que en Morelos el conocimiento que los/las adolescentes tienen sobre la $\mathrm{AE}$ es escaso. Futuros estudios podrían indagar en busca de explicaciones sobre este hecho, $\mathrm{y}$, particularmente, se debe explorar si el conocimiento incorrecto que existe sobre $\mathrm{AE}$ es consecuencia de que la difusión de la información tiene un alcance limitado entre esta población, o bien, a que la información que se difunde resulta confusa. Otra posibilidad es que los mensajes sobre un uso no adecuado de la AE provengan directamente de ciertas fuentes de información como los pares o adultos mal informados.

Resulta necesario transmitir a los/las adolescentes mensajes claros sobre la forma precisa de uso de este método y sus indiscutibles ventajas, así como proveerles de información verídica respecto del funcionamiento biológico de la $\mathrm{AE}$. Esto permitiría disminuir nociones de orden moral y desmitificar el concepto de la $\mathrm{AE}$ como un método abortivo, favoreciendo así su uso. Se requiere identificar las vías que están resultando efectivas en la promoción de este método, así como reforzar su promoción y distribución dentro de programas ya establecidos como los llamados "servicios amigables" para los/las adolescentes, pues esto podría favorecer el diseño de estrategias efectivas para prevenir embarazos no planeados entre ellos. 


\section{Referencias}

I. Secretaría de Salud. Norma Oficial Mexicana NOM-005-SSA2-1993, Servicios de Planificación Familiar. Diario Oficial de la Federación, 2I de enero de 2004

2. Secretaría de Salud.Actualización Cuadro Básico de Medicamentos del Sector Salud. Diario Oficial de la Federación, II de julio de 2005. 3. Schiavon ER.Adolescentes y anticoncepción. Gac Med Mex 2006;Supl 2(142): III-116.

4. Schiavon ER, Jiménez CH, Ellertson Ch, Langer A. Anticoncepción de emergencia: Un método simple, seguro, efectivo y económico para prevenir embarazos no deseados. Rev Invest Clin 2000;52(2): 168- I76. 5. Núñez-Urquiza RM, Hernández-Prado B, García-Barrios C, González D,Walker D. Embarazo no deseado en adolescentes y utilización de métodos anticonceptivos posparto. Salud Publica Mex 2003;Suppl I:S92-SI02.

6. Webb A. How safe is the Yuzpe method of emergency contraception? Fert Control Rev 1995;4: 16-18. 7. Rodriguez I, Grou F, Joly J. Effectiveness of emergency contraceptive pills between 72 and 120 hours after unprotected intercourse.Am J Obstet Gynecol 200 I; 184:531-537. 7. Rodríguez I, Grou F, Joly J. Effectiveness of emergency contraceptive pills between 72 and I20 hours after unprotected intercourse. Am J Obstet Gynecol 2001;184:531-537.

8. Webb A, Taberner D. Clotting factors after emergency contraception. Adv Contracep 1993;9:75-82.

9. Trussell J, Stewart F, Guest F, Hatcher RA. Emergency contraceptive pills: a simple proposal to reduce unintended pregnancies. Fam Plann Perspect 1992;24:269-273.

10. Comparetto C, Giudici S, Coccia ME, Scarselli G. Clinical, ethical, and medical legal considerations on emergency contraception. Clin Exp Obstet Gynecol 2005;32(2): 107-I I0.

II. Haynes KA.An update on emergency contraception: Use in Adolescents. J Pediatr Nurs 2007;22(3): 186-195.

12. Foster DG, Ralph LJ,Arons A, Brindis CD, Harper CC. Trends in knowledge of emergency contraception Among women in California, 1999-2004. Womens Health Issues 2007; 17:22-28.

13. Shoveller J, Chabot C, Soon JA, Levine M. Identifying Barriers to Emergency Contraception Use Among Young Women from Various Sociocultural Groups in British Columbia, Canada. Perspect Sex Reprod Health 2007;39(I):13-20.

14. Aiken AM, Gold MA, Parker AM. Changes in Young Women's Awareness, Attitudes, and Perceived Barriers to Using Emergency Contraception.J Pediatr Adolesc Gynecol 2005; 18(I):25-32.

15. Frenk J.Tender puentes: lecciones globales desde México sobre políticas de salud basadas en evidencias. Salud Publica Mex 2007;49 supl I:SI4-S22.

16. Partida-Bush V. Situación demográfica nacional y estatal. México: Consejo Nacional de Población, 2006.

17. Muñoz-Aguirre CD. Cambio demográfico y desarrollo social de los jóvenes. México: Consejo Nacional de Población, 2006.

18. Consejo Nacional de Población, 2006. Comunicado de prensa 34/06. México, DF,Viernes II de agosto de 2006.

19. Secretaría de Salud/ Instituto Nacional de Salud Pública. Encuesta Nacional de Salud y Nutrición. México: Ssa/INSP 2006.

20. INEGI. Dirección General de Estadística. Dirección de Análisis y Estudios Demográficos. Cuaderno I6, 2004. México: INEGI, 2004

21. Instituto Mexicano de la Juventud. Encuesta Nacional de Juventud 2005. Módulo Sexualidad. México, D.F.: Instituto Mexicano de la Juventud. 2006.
22. Hatcher RA, Trussell J, Stewart F, Howells S, Russell CR, Kowal D. Emergency Contraception:The Nation's Best Kept Secret. In: Decatur GA. Bridging the Gap Communications. Atlanta, 1995.

23. Braeken D, Otoo-Oyortey N, Serour G.Access to sexual and reproductive health care:Adolescents and young people. Int J Gynaecol Obstet 2007;98:172-174.

24. United Nations Population Fund.Valorizar a 1000 millones de adolescentes: inversiones en su salud y sus derechos. New York: UNFPA, 2003.

25. Lazcano-Ponce EC, Hernández B, Cruz-Valdez A, Allen B, Díaz $R$, Hernández $C$, et al. Chronic disease risk factors among healthy adolescents attending public schools in the state of Morelos, Mexico.Arch Med Res 2003;34(3):222-236.

26. Bronfman M, Guiscafré H, Castro R, Gutiérrez G. Medición de la desigualdad: una estrategia metodológica, análisis de las características socioeconómicas de la muestra. Arch Invest Med 1988;19:351-360. 27. Verduzco MA, Lara MA,Acevedo M.Validez del inventario de autoestima de Coopersmith para niños mexicanos. Revista Intercontinental de Psicología 1994;7:55-64

28. Hosmer DW, Lemeshow S.Applied logistic regression. Nueva York: John Wiley \& Sons, 1989.

29. Harvey SM, Beckman LJ, Sherman C, Petitti D. Women's Experience and Satisfaction with Emergency Contraception. Fam Plann Perspect 1999;3I(5):237-240, 260.

30. Walker D, Torres P, Gutiérrez JP, Flemming K, Bertozzi S. Emergency Contraception Use Is Correlated with Increased Condom Use among Adolescents: Results from Mexico. Journal of Adolescent Health 2004;35:329-334.

3I. Menkes C, Suárez L, Núñez L, González S. La salud reproductiva de los estudiantes de educación secundaria y media superior de Chiapas, Guanajuato, Guerrero, Puebla y San Luis Potosí. México: UNAM/CRIM, 2006. 32. Ekstrand M,Tydén T, Darj E, Larsson M. Preventing pregnancy: a girls' issue. Seventeen-year-old Swedish boys perceptions on abortion, reproduction and use of contraception. Eur J Contracept Reprod Health Care 2007; 12(2): I I - I I8.

33. De Keijzer B. Los hombres ante la salud sexual y reproductiva: una relación contradictoria. En: Bronfman M, Denmac C. Salud reproductiva: Temas y debates. México: Instituto Nacional de Salud Reproductiva, 2003. 34. Manlove J, Ryan S, Franzetta K. Patterns of contraceptive use within teenagers' first sexual relationships. Perspect Sex Reprod Health. 2003;35(6):246-255.

35. Merkh RD,Whittaker PG, Baker K, Hock-Long L,Armstrong K.Young unmarried men's understanding of female hormonal contraception. Contraception 2009;79(3):228-235.

36. Menkes C, Suarez L. Sexualidad y Embarazo Adolescente en México. Papeles de Población 2003;35:233-263.

37. Tapia-Curiel A,Villaseñor-Farías M, Nuño-Gutiérrez BL. Knowledge and attitudes about using emergency contraceptives among young college students. Rev Med Inst Mex Seguro Soc 2008;46(I):33-4I.

38. Juárez F, Gayet C. Salud Sexual y reproductiva de los adolescentes en México: un nuevo marco de análisis para la evaluación y diseño de políticas. Papeles de Población 2005; I I (045): I77-219.

39. Hernández B,Velasco-Mondragón HE. Encuestas transversales. Salud Publica Mex 2000;42(5):447-455.

40. Hickey MT. Female college students' knowledge, perceptions, and use of emergency contraception.J Obstet Gynecol Neonatal Nurs 2009;38(4):399-405.

4I. Heimburger A,Acevedo-Garcia D, Schiavon R, Langer A, Mejia G, Corona G, et al. Emergency contraception in Mexico City: knowledge, attitudes, and practices among providers and potential clients after a 3-year introduction effort. Contraception 2002;66(5):321-329. 